\title{
Signal Transduction Induction
}

National Cancer Institute

\section{Source}

National Cancer Institute. Signal Transduction Induction. NCI Thesaurus. Code C4082O.

Signal T ransduction Induction involves the initiation of activity in cellular processes by which a cell propagates information from an extracellular signal through a series of biochemical reactions inside the cell to produce biological responses to events in the environment or internal milieu. 